\title{
Rigorous Proof of Cubic Convergence for the dqds Algorithm for Singular Values
}

\author{
Kensuke Aishima, Takayasu Matsuo and Kazuo Murota
}

Department of Mathematical Informatics

Graduate School of Information Science and Technology

University of Tokyo

E-mail: Kensuke_Aishima@mist.i.u-tokyo.ac.jp

Received March 27, 2007

Revised May 14, 2007

\begin{abstract}
Fernando and Parlett observed that the dqds algorithm for singular values can be made extremely efficient with Rutishauser's choice of shift; in particular it enjoys "local" (or one-step) cubic convergence at the final stage of iteration, where a certain condition is to be satisfied. Their analysis is, however, rather heuristic and what has been shown is not sufficient to ensure asymptotic cubic convergence in the strict sense of the word. The objective of this paper is to specify a concrete procedure for the shift strategy and to prove with mathematical rigor that the algorithm with this shift strategy always reaches the "final stage" and enjoys asymptotic cubic convergence.
\end{abstract}

Key words: singular value, bidiagonal matrix, dqds algorithm

\section{Introduction}

Every $n \times m$ real matrix $A$ of rank $r$ can be decomposed into

$$
A=U \Sigma V^{\mathrm{T}}
$$

with suitable orthogonal matrices $U \in \mathbf{R}^{n \times n}$ and $V \in \mathbf{R}^{m \times m}$, where

$$
\Sigma=\left(\begin{array}{cc}
D & O_{r, m-r} \\
O_{n-r, r} & O_{n-r, m-r}
\end{array}\right), \quad D=\operatorname{diag}\left(\sigma_{1}, \ldots, \sigma_{r}\right),
$$

and $\sigma_{1} \geq \cdots \geq \sigma_{r}>0$. The notation $O_{k, l}$ means a $k \times l$ zero matrix. The nonzero diagonal elements $\sigma_{1}, \ldots, \sigma_{r}$ are the singular values of $A$, which play important roles in application areas. Accordingly, numerical methods for computing singular values are of great importance in practice.

The singular values of $A$ are equal to the square roots of the eigenvalues of $A^{\mathrm{T}} A$ and hence an iterative computation is inevitable for singular values. Usually, the given matrix $A$ is first transformed to a bidiagonal matrix to reduce the overall computational cost. In the case of $n \geq m$, for example, the matrix $A$ can be transformed, with appropriate orthogonal matrices $\tilde{U} \in \mathbf{R}^{n \times n}$ and $\tilde{V} \in \mathbf{R}^{m \times m}$, as

$$
\tilde{U}^{\mathrm{T}} A \tilde{V}=\left(\begin{array}{c}
B \\
O_{n-m, m}
\end{array}\right),
$$

where $B \in \mathbf{R}^{m \times m}$ is an upper bidiagonal matrix. The singular values of $B$ coincide with those of $A$. 
Most of the current methods for computing singular values of bidiagonal matrices are based on the QR algorithm [4]. Demmel and Kahan's improvement [5] on the QR algorithm, which was awarded the second SIAM prize in numerical linear algebra, is available as DBDSQR in LAPACK [3, 11].

In the study of this algorithm, the differential quotient difference (dqd) algorithm was proposed by Fernando-Parlett [9], with subsequent introduction of shifts to accelerate the convergence. This algorithm is now called the differential quotient difference with shifts (dqds) algorithm. The dqds algorithm has been popular due to its accuracy, speed and numerical stability, and is implemented as DLASQ in LAPACK $[3,11,14]$. The dqds is integrated into Multiple Relatively Robust Representations $\left(\mathrm{MR}^{3}\right)$ algorithm $[6,7,8]$. It may also be said that the dqds algorithm is a numerically stabler version of the pqds algorithm, which in turn is the pqd algorithm [10] with shifts incorporated to accelerate the convergence.

As for theoretical analysis about the dqds algorithm, locally quadratic or cubic convergence has been discussed in [9] under certain assumptions. In [9], global convergence of the dqds has also been discussed using ideas from [15] under several restrictions. A recent paper of the present authors [1] describes the present status of the global convergence theorems and gives a general theorem for global convergence. The paper has also revealed the asymptotic rate of 1.5 for the Johnson shift. It is pointed out in [2] that superquadratic convergence can be realized with a simple shift strategy.

The objective of this paper is to give a mathematically rigorous proof for the asymptotic cubic convergence of the dqds algorithm that employs the shift strategy proposed by Fernando-Parlett based on Rutishauser's idea. More specifically, this paper is organized as follows. In Section 2 the target problem is defined. Section 3 is devoted to summarizing the dqds algorithm. In Section 4 the basic facts on convergence of the dqds are summarized based on [1]. The existing results for local cubic convergence are reviewed in Section 5.1 with emphasis on what additional argument is required in order to conclude asymptotic cubic convergence in the strict sense of the word. Then in Section 5.2 a concrete procedure for the shift is proposed, and in Section 6 it is shown that with the shift procedure the dqds always realizes asymptotic cubic convergence.

\section{Problem setting}

We assume that the given real matrix $A$ has already been transformed to a bidiagonal matrix

$$
B=\left(\begin{array}{cccc}
b_{1} & b_{2} & & \\
& b_{3} & \ddots & \\
& & \ddots & b_{2 m-2} \\
& & & b_{2 m-1}
\end{array}\right),
$$

to which the dqds algorithm is applied. 
Following [9], we assume

Assumption (A). The bidiagonal elements of $B$ are positive, i.e., $b_{k}>0$ for $k=1,2, \ldots, 2 m-1$.

This assumption guarantees (see [13]) that the singular values of $B$ are all distinct: $\sigma_{1}>\cdots>\sigma_{m}>0$.

Assumption (A) is not restrictive, in theory or in practice. In fact, if a subdiagonal element is zero, i.e., $b_{2 k}=0$ for some $k$, then the problem reduces to two independent problems on matrices of smaller sizes, $k \times k$ and $(m-k) \times(m-k)$. If there is a zero element on the diagonal, several iterations of the dqd algorithm (i.e., the dqds algorithm without shifts) suffice to remove the diagonal zero, and the problem is again separated into a set of smaller problems (see [9] for details). Finally, it is easy to see that the singular values are invariant if $b_{k}$ is replaced by $\left|b_{k}\right|$.

In our problem setting we have assumed real matrices, whereas the singular value decomposition is also defined for complex matrices. Our restriction to real matrices is justified by the fact that any complex matrix can be transformed to a real bidiagonal matrix by, say, (complex) Householder transformations, while keeping its singular values [9].

\section{The dqds algorithm}

The dqds algorithm can be described in computer program form as follows.

Algorithm 3.1. The dqds algorithm

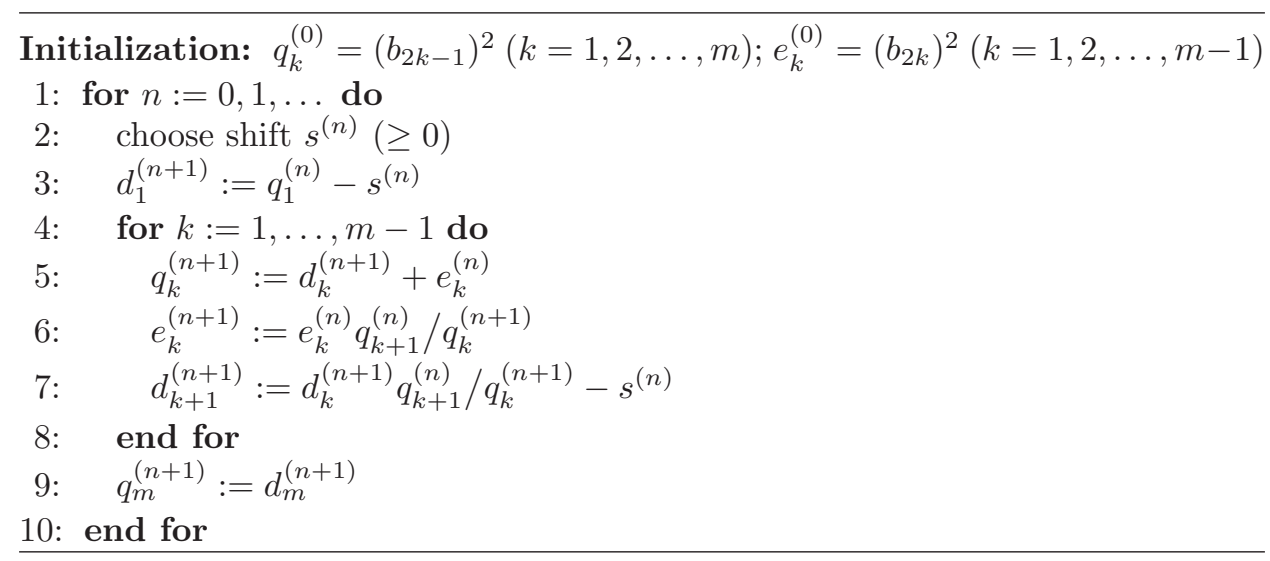

The outermost loop is terminated when some suitable convergence criterion, say, $\left|e_{m-1}^{(n)}\right| \leq \epsilon$ for some prescribed constant $\epsilon>0$, is satisfied. At the termination we have

$$
\sigma_{m}^{2} \approx q_{m}^{(n)}+\sum_{l=0}^{n-1} s^{(l)}
$$


and hence $\sigma_{m}$ can be approximated by $\sqrt{q_{m}^{(n)}+\sum_{l=0}^{n-1} s^{(l)}}$. Then by the deflation process the problem is shrunk to an $(m-1) \times(m-1)$ problem, and the same procedure is repeated until $\sigma_{m-1}, \ldots, \sigma_{1}$ are obtained in turn.

It turns out to be convenient to introduce additional notations $e_{0}^{(n)}$ and $e_{m}^{(n)}$ with "boundary conditions":

$$
e_{0}^{(n)}=0, \quad e_{m}^{(n)}=0 \quad(n=0,1, \ldots)
$$

to simplify the expression of the algorithm. Let

$$
B^{(n)}=\left(\begin{array}{cccc}
b_{1}^{(n)} & b_{2}^{(n)} & & \\
& b_{3}^{(n)} & \ddots & \\
& & \ddots & b_{2 m-2}^{(n)} \\
& & & b_{2 m-1}^{(n)}
\end{array}\right)
$$

$b_{k}^{(0)}=b_{k}(k=1,2, \ldots, 2 m-1)$, and

$$
\begin{aligned}
& q_{k}^{(n)}=\left(b_{2 k-1}^{(n)}\right)^{2} \quad(k=1,2, \ldots, m ; n=0,1, \ldots), \\
& e_{k}^{(n)}=\left(b_{2 k}^{(n)}\right)^{2} \quad(k=1,2, \ldots, m-1 ; n=0,1, \ldots) .
\end{aligned}
$$

Then Algorithm 3.1 can be rewritten in terms of the Cholesky decomposition (with shifts):

$$
\left(B^{(n+1)}\right)^{\mathrm{T}} B^{(n+1)}=B^{(n)}\left(B^{(n)}\right)^{\mathrm{T}}-s^{(n)} I,
$$

where $B^{(0)}=B$. In other words, a single step of the dqds algorithm $\left(\left\{q_{k}^{(n)}\right\},\left\{e_{k}^{(n)}\right\}\right) \mapsto\left(\left\{q_{k}^{(n+1)}\right\},\left\{e_{k}^{(n+1)}\right\}\right)$ is equivalent to a single step of the shifted Cholesky LR method $B^{(n)}\left(B^{(n)}\right)^{\mathrm{T}} \mapsto B^{(n+1)}\left(B^{(n+1)}\right)^{\mathrm{T}}$, where

$$
\begin{aligned}
& B^{(n)}\left(B^{(n)}\right)^{\mathrm{T}}
\end{aligned}
$$

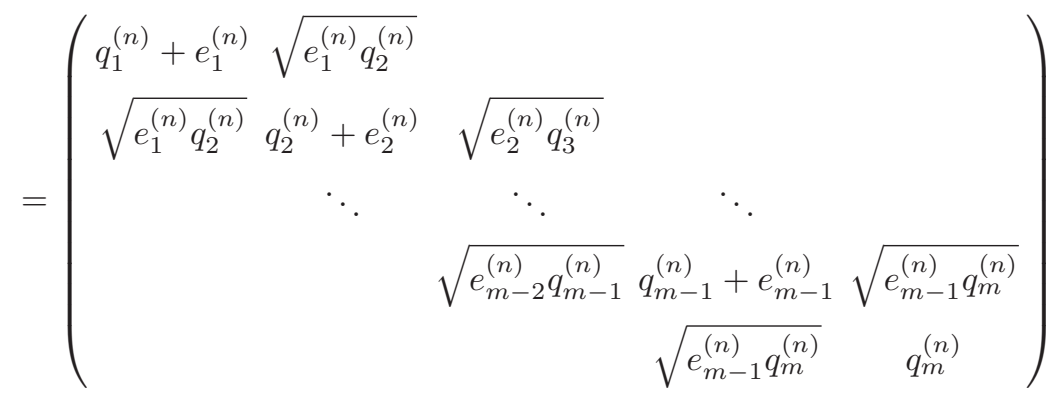

can be easily seen from (3), (4), and (5). From (6) it follows that

$$
\left(B^{(n)}\right)^{\mathrm{T}} B^{(n)}=W^{(n)}\left(\left(B^{(0)}\right)^{\mathrm{T}} B^{(0)}-\sum_{l=0}^{n-1} s^{(l)} I\right)\left(W^{(n)}\right)^{-1},
$$


where $W^{(n)}=\left(B^{(n-1)} \cdots B^{(0)}\right)^{-\mathrm{T}}$ is a nonsingular matrix. Therefore the eigenvalues of $\left(B^{(n)}\right)^{\mathrm{T}} B^{(n)}$ are the same as those of $\left(B^{(0)}\right)^{\mathrm{T}} B^{(0)}-\sum_{l=0}^{n-1} s^{(l)} I$.

If $s^{(n)}<\left(\sigma_{\text {min }}^{(n)}\right)^{2}$ in each iteration $n$, where $\sigma_{\text {min }}^{(n)}$ is the smallest singular value of $B^{(n)}, B^{(n)}$ converges to a diagonal matrix as $n \rightarrow \infty$, and then, by (8), the singular values of $B$ can be obtained from the diagonal elements of $B^{(n)}$ with sufficiently large $n$ (see Theorem 4.1). Moreover, if $s^{(n)}<\left(\sigma_{\min }^{(n)}\right)^{2}$, the variables in the dqds algorithm are always positive so that the algorithm does not break down (see Lemma 4.1).

\section{Fundamental facts about convergence}

Some relevant facts about the dqds algorithm are reviewed in this section. We begin with the fundamental convergence theorem. Recall that $\sigma_{1}>\sigma_{2}>\cdots>\sigma_{m}$ are singular values of $B$ and $\sigma_{\text {min }}^{(n)}$ denotes the smallest singular value of $B^{(n)}$.

THEOREM 4.1 (Convergence of the dqds algorithm [1]). Suppose the matrix $B$ satisfies Assumption (A), and the shift in the dqds algorithm satisfies

$$
0 \leq s^{(n)}<\left(\sigma_{\min }^{(n)}\right)^{2}
$$

for all $n=0,1,2, \ldots$ Then

$$
\sum_{n=0}^{\infty} s^{(n)} \leq \sigma_{m}^{2}
$$

Moreover,

$$
\begin{aligned}
& \lim _{n \rightarrow \infty} e_{k}^{(n)}=0 \quad(k=1,2, \ldots, m-1), \\
& \lim _{n \rightarrow \infty} q_{k}^{(n)}=\sigma_{k}^{2}-\sum_{n=0}^{\infty} s^{(n)} \quad(k=1,2, \ldots, m) .
\end{aligned}
$$

In matrix form, we have

$$
\lim _{n \rightarrow \infty}\left(B^{(n)}\right)^{\mathrm{T}} B^{(n)}=\operatorname{diag}\left(\sigma_{1}^{2}-\sum_{n=0}^{\infty} s^{(n)}, \ldots, \sigma_{m}^{2}-\sum_{n=0}^{\infty} s^{(n)}\right) .
$$

The variables are guaranteed to remain positive, as follows. This fact is crucial to the proof of the convergence theorems as well as to numerical stability of the algorithm.

Lemma 4.1. Suppose the dqds algorithm is applied to the matrix $B$ satisfying Assumption (A). If $s^{(l)}<\left(\sigma_{\text {min }}^{(l)}\right)^{2}$ for $l=0,1, \ldots, n$, then $\left(B^{(l+1)}\right)^{\mathrm{T}} B^{(l+1)}$ are positive definite for $l=0,1, \ldots, n$, and hence $q_{k}^{(l+1)}>0(k=1, \ldots, m), e_{k}^{(l+1)}>0$ $(k=1, \ldots, m-1)$, and $d_{k}^{(l+1)}>0(k=1, \ldots, m)$ for $l=0,1, \ldots, n$. 
Proof. For completeness we give a proof based on [1]. The proof is by induction on $n$. Under Assumption (A), we have $q_{k}^{(0)}>0, e_{k}^{(0)}>0$ and that $\left(B^{(0)}\right)^{\mathrm{T}} B^{(0)}$ is positive definite. Suppose that $\left(B^{(n)}\right)^{\mathrm{T}} B^{(n)}$ is positive definite and $q_{k}^{(n)}>0, e_{k}^{(n)}>0$. By $(6)$, if $s^{(n)}<\left(\sigma_{\min }^{(n)}\right)^{2}$, then $\left(B^{(n+1)}\right)^{\mathrm{T}} B^{(n+1)}$ is positive definite because $B^{(n)}\left(B^{(n)}\right)^{\mathrm{T}}-s^{(n)} I$ is positive definite. Therefore all the diagonal elements of $B^{(n+1)}$ are nonzero $\left(b_{2 k-1}^{(n+1)} \neq 0\right)$ and hence $q_{k}^{(n+1)}>0$ because of (4). By the 6th line of Algorithm 3.1, we have $e_{k}^{(n+1)}>0$. The inequality $d_{k}^{(n+1)}>0$ is proved by contradiction as follows. If we had $d_{k}^{(n+1)} \leq 0$ for some $k$, we would have $d_{k+1}^{(n+1)} \leq 0$ by the 7 th line of Algorithm 3.1 and then $q_{m}^{(n+1)}=d_{m}^{(n+1)} \leq 0$. This contradicts $q_{m}^{(n+1)}>0$.

The asymptotic rate of convergence of the dqds algorithm is given by the following lemma.

Lemma 4.2 ([1]). Under the same assumption as in Theorem 4.1, we have

$$
\lim _{n \rightarrow \infty} \frac{e_{k}^{(n+1)}}{e_{k}^{(n)}}=\frac{\sigma_{k+1}^{2}-\sum_{n=0}^{\infty} s^{(n)}}{\sigma_{k}^{2}-\sum_{n=0}^{\infty} s^{(n)}}<1 \quad(k=1, \ldots, m-1) .
$$

Therefore, $e_{k}^{(n)}(k=1, \ldots, m-2)$ are of linear convergence as $n \rightarrow \infty$. The bottommost element $e_{m-1}^{(n)}$ shows superlinear convergence if $\sigma_{m}^{2}-\sum_{n=0}^{\infty} s^{(n)}=0$.

The following lemma will be used in the proof of Lemma 6.1 that shows the validity of our shift strategy to be described in Section 5.2.

LEMMA 4.3. For a fixed $n$, assume $e_{k}^{(n)}>0(k=1, \ldots, m-1)$ and $q_{k}^{(n)}>0$ $(k=1, \ldots, m)$, and apply Algorithm 3.1 with shift $s^{(n)}$ to compute $d_{k}^{(n+1)}(k=$ $1, \ldots, m)$. Then $s^{(n)}<\left(\sigma_{\min }^{(n)}\right)^{2}$ if and only if $d_{k}^{(n+1)}>0(k=1, \ldots, m)$.

Proof. First suppose that $d_{k}^{(n+1)}>0(k=1, \ldots, m)$. From the 5 th line of Algorithm 3.1 we have $q_{k}^{(n+1)}>0(k=1, \ldots, m)$. Then the diagonal elements of $B^{(n+1)}$ are positive by (4). Furthermore, by the 6th line of Algorithm 3.1 we see $e_{k}^{(n+1)}>0(k=1, \ldots, m-1)$, and hence $B^{(n+1)}$ is a real matrix by $(5)$. Therefore $\left(B^{(n+1)}\right)^{\mathrm{T}} B^{(n+1)}$ is positive definite, and hence we have $s^{(n)}<\left(\sigma_{\min }^{(n)}\right)^{2}$ from (6).

Conversely suppose that $s^{(n)}<\left(\sigma_{\min }^{(n)}\right)^{2}$ is true. Then by (6) we have $b_{2 k-1}^{(n+1)} \neq 0$ $(k=1, \ldots, m)$, which are diagonal elements of $B^{(n+1)}$. Therefore we have $q_{k}^{(n+1)}>0$ $(k=1, \ldots, m)$ from (4). By the 6th line of Algorithm 3.1, we see $e_{k}^{(n+1)}>0$ $(k=1, \ldots, m-1)$. The inequality $d_{k}^{(n+1)}>0$ is proved by contradiction as follows. If we had $d_{k}^{(n+1)} \leq 0$ for some $k$, we would have $d_{k+1}^{(n+1)} \leq 0$ by the 7 th line of Algorithm 3.1 and then $q_{m}^{(n+1)}=d_{m}^{(n+1)} \leq 0$ from the 9th line of Algorithm 3.1. This is a contradiction. 
Note that the assumption of Lemma 4.3 is satisfied for $n=0$ by Assumption (A). By Lemma 4.1 the assumption of Lemma 4.3 will be met for all $n$ if the condition (9) is satisfied. Theorem 4.1, on the other hand, guarantees the convergence, whereas Lemma 4.2 shows that the convergence rate is at least linear.

\section{Shift for cubic convergence}

In this section, we first briefly review the existing results for cubic convergence and discuss what is still missing in those results (Section 5.1). Then in Section 5.2 we propose a concrete procedure for the shift.

\subsection{Review of the existing results}

The history of search for a shift yielding cubic convergence dates back to Rutishauser [15], showing that the Cholesky LR method with a certain shift strategy is expected to converge cubically when applied to positive definite symmetric matrices. The idea has been generalized later in several different ways [16] (see also the discussion in $[9, \S 8.3])$. In this subsection, we review the idea in the context of our problem setting, and clarify what additional argument should be made before we can obtain a rigorous mathematical proof of asymptotic cubic convergence.

Let us introduce some notations. We split $B^{(n)}\left(B^{(n)}\right)^{\mathrm{T}}$ in $(7)$ into blocks as

$$
B^{(n)}\left(B^{(n)}\right)^{\mathrm{T}}=\left(\begin{array}{cc}
U^{(n)} & \boldsymbol{v}^{(n)} \\
\left(\boldsymbol{v}^{(n)}\right)^{\mathrm{T}} & q_{m}^{(n)}
\end{array}\right),
$$

where $U^{(n)}$ is an $(m-1) \times(m-1)$ matrix and $\boldsymbol{v}^{(n)}=\left(0, \ldots, 0, \sqrt{e_{m-1}^{(n)} q_{m}^{(n)}}\right)^{\mathrm{T}}$. The eigenvalues of $U^{(n)}$ are denoted by $\lambda_{i}^{(n)}(i=1, \ldots, m-1)$. Recall that a single step of the dqds algorithm is equivalent to a single step of the shifted Cholesky LR applied to the matrix $B^{(n)}\left(B^{(n)}\right)^{\mathrm{T}}$. Also note that under Assumption (A), the eigenvalues of $B^{(n)}\left(B^{(n)}\right)^{\mathrm{T}}$ are all distinct, and the subdiagonal elements of $B^{(n)}\left(B^{(n)}\right)^{\mathrm{T}}$ are all positive.

Now suppose that $n$ is sufficiently large and the matrix $B^{(n)}\left(B^{(n)}\right)^{\mathrm{T}}$ is at the final stage of convergence. More specifically, we assume that the following condition is satisfied:

$$
\lambda_{i}^{(n)}-q_{m}^{(n)}>g \quad(i=1, \ldots, m-1),
$$

where $g$ is a constant independent of $n$ satisfying $0<g<\sigma_{m-1}^{2}-\sigma_{m}^{2}$.

Rutishauser suggested the following shift strategy to modify $B^{(n)}$ to $B^{(n+1)}$. Under the condition (14), the matrix $U^{(n)}-q_{m}^{(n)} I$ is positive definite, whereas the smallest eigenvalue of $B^{(n)}\left(B^{(n)}\right)^{\mathrm{T}}-q_{m}^{(n)} I$ is negative by Assumption (A). This implies that if we apply the algorithm of Cholesky factorization to $B^{(n)}\left(B^{(n)}\right)^{\mathrm{T}}-$ $q_{m}^{(n)} I$, it must fail at the very last stage with a negative number, say $\hat{q}_{m}^{(n+1)}<0$, remaining at the lower right diagonal position. Note that $\hat{q}_{m}^{(n+1)}$ is nothing but the 
$q_{m}^{(n+1)}$ obtained by a single step of the dqds algorithm with the shift $q_{m}^{(n)}$. This $\hat{q}_{m}^{(n+1)}$ can be utilized to modify the "tentative" shift $q_{m}^{(n)}$ to an eligible shift as follows.

\section{RUTISHAUSER'S SHIFT STRATEGY.}

1. Try the Cholesky factorization of $B^{(n)}\left(B^{(n)}\right)^{\mathrm{T}}-q_{m}^{(n)} I$ to find the value $\hat{q}_{m}^{(n+1)}<0$.

2. Do a shifted Cholesky LR step (or equivalently, a dqds step) with the shift $q_{m}^{(n)}+\hat{q}_{m}^{(n+1)}$ to obtain $B^{(n+1)}\left(B^{(n+1)}\right)^{\mathrm{T}}$.

The trick in this strategy is that the shift $q_{m}^{(n)}+\hat{q}_{m}^{(n+1)}$ is always valid so that the shifted Cholesky LR in the step 2 is feasible.

Lemma 5.1 ([15, Th. 1], $[16, \S 56])$. If the condition (14) is satisfied at step n, then $q_{m}^{(n)}+\hat{q}_{m}^{(n+1)}<\left(\sigma_{\min }^{(n)}\right)^{2}$.

The next theorem shows the "local" cubic convergence. By saying "local" we intend to emphasize that the inequality (15) is claimed to be true for a particular $n$, and not for all sufficiently large $n$.

Theorem 5.1 (Local cubic convergence [15, Th. 4], [16, §57]). If Rutishauser's strategy is used to obtain $B^{(n+1)}\left(B^{(n+1)}\right)^{\mathrm{T}}$ at step $n$ at which the condition (14) is satisfied, then we have

$$
\frac{e_{m-1}^{(n+1)} q_{m}^{(n+1)}}{g^{2}} \leq\left(\frac{e_{m-1}^{(n)} q_{m}^{(n)}}{g^{2}}\right)^{3}
$$

That is, the lowermost subdiagonal element of $B^{(n)}\left(B^{(n)}\right)^{\mathrm{T}}$ decays cubically in this single step.

Our aim in this paper is to provide a rigorous proof of asymptotic cubic convergence of the dqds. Theorem 5.1 is not eligible for this purpose in the following senses. First, for "asymptotic" cubic convergence, the condition (14) must be satisfied continuously (or consecutively) for all sufficiently large $n$. This is, however, not guaranteed but assumed in Theorem 5.1. Second, Theorem 5.1 does not say anything about how the dqds iteration can be led to its "final stage of convergence" for an arbitrary matrix $B$. This issue is closely related to the problem of global convergence. A proof of global convergence has been given in [9] in the absence of shifts. With this result, we see that if the dqds is run without shifts, the condition (14) is eventually satisfied. The rate of convergence with this strategy, however, stays only linear in the early phase; even worse, once the shift for cubic convergence becomes active, the global convergence is no longer guaranteed by the global convergence theorem given in [9]. Another proof of global convergence has been given in [15], which covers more general shifts. The theorem, however, applies to nondegenerate cases only, and in this respect it is not sufficient for our purpose. 
The global convergence theorem (Theorem 4.1), which is valid for any shifts satisfying $0 \leq s^{(n)}<\left(\sigma_{\min }^{(n)}\right)^{2}$ and for an arbitrary matrix $B$, allows us to employ more aggressive shift strategies from the early phase of iteration, while keeping the theoretical guarantee of global convergence.

In the subsequent sections, we propose a concrete shift strategy based on Rutishauser's, and show that with the strategy the condition (14) is continuously satisfied (Lemma 6.2), and the cubic convergence is in fact realized (Theorem 6.1).

REMARK 5.1. In the dqds algorithm, the computation in the first step of Rutishauser's shift strategy can be simplified as follows [9, $\S 8.3]$. If we substitute the 5th line of Algorithm 3.1 into the 7 th line, we obtain

$$
d_{k+1}^{(n+1)}=\frac{d_{k}^{(n+1)} q_{k+1}^{(n)}}{d_{k}^{(n+1)}+e_{k}^{(n)}}-s^{(n)} .
$$

As a special case, with the tentative shift $s^{(n)}=q_{m}^{(n)}$ we have

$$
\hat{d}_{k+1}^{(n+1)}=\frac{\hat{d}_{k}^{(n+1)} q_{k+1}^{(n)}}{\hat{d}_{k}^{(n+1)}+e_{k}^{(n)}}-q_{m}^{(n)} .
$$

(The hat "^" indicates that the quantity is obtained with the tentative shift $s^{(n)}=$ $\left.q_{m}^{(n)}\right)$. Thus we can concentrate on the computation of $\hat{d}_{k}^{(n+1)}(k=1, \ldots, m)$ to obtain $\hat{q}_{m}^{(n+1)}=\hat{d}_{m}^{(n+1)}$, while $\hat{q}_{k}^{(n+1)}$ and $\hat{e}_{k}^{(n+1)}$ (accordingly the 5 th and the 6 th line of Algorithm 3.1) can be skipped. In this way the cost to determine $\hat{q}_{m}^{(n+1)}$ is "about $2 / 3$ of a dqds step" [9].

\subsection{Concrete procedure for the shift}

In this section we realize Rutishauser's idea as a concrete procedure to choose a shift.

The procedure goes as follows. At each iteration step $n$ we first compute $\hat{d}_{k}^{(n+1)}$ as in Section 5.1. If $\hat{d}_{k}^{(n+1)}>0$ for $k=1, \ldots, m-1$, we set the shift $s^{(n)}$ as

$$
s^{(n)}=\frac{\hat{d}_{m-1}^{(n+1)} q_{m}^{(n)}}{\hat{d}_{m-1}^{(n+1)}+e_{m-1}^{(n)}} ;
$$

otherwise we put $s^{(n)}=0$. It is easy to verify that the shift given by (18) coincides with $s^{(n)}=q_{m}^{(n)}+\hat{q}_{m}^{(n+1)}$ in Rutishauser's shift strategy (see Remark 5.1 and the equation (17) therein) for which the "local" cubic convergence is already discussed in Section 5.1.

A formal description of this procedure is given below under the name of "shift strategy (C)". 


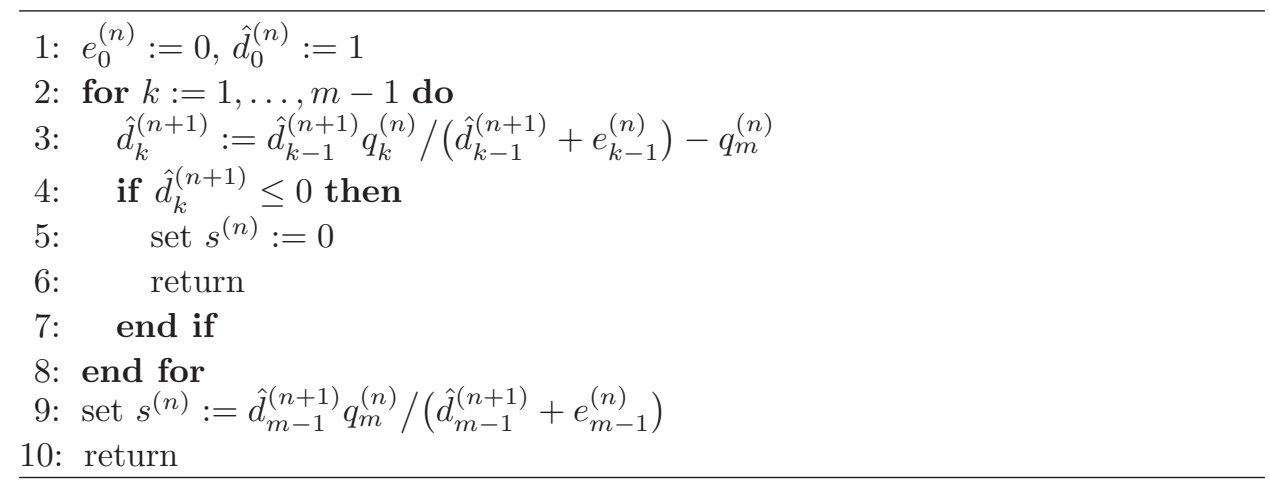

In the next section, we show that the shift determined by the above procedure is a valid choice, satisfying the condition (9) in Theorem 4.1. By Lemma 4.1 this guarantees positivity of the variables, which in turn implies that the algorithm is free from breakdown. Furthermore we shall establish a theorem of asymptotic cubic convergence with mathematical rigor.

\section{Theorem of cubic convergence}

In this section, we prove that the asymptotic cubic convergence is realized by the shift strategy $(\mathrm{C})$ described in Section 5.2. The proof consists of showing the following facts.

(i) The condition $0 \leq s^{(n)}<\left(\sigma_{\min }^{(n)}\right)^{2}$ of (9) is always satisfied (Lemma 6.1).

(ii) The shift is given by $s^{(n)}=\hat{d}_{m-1}^{(n+1)} q_{m}^{(n)} /\left(\hat{d}_{m-1}^{(n+1)}+e_{m-1}^{(n)}\right)$ for all sufficiently large $n$ (Lemma 6.2).

(iii) Asymptotic cubic convergence is realized (Theorem 6.1).

The following fundamental fact is known in the literature [9]; we give here an alternative proof in a way consistent with our approach.

LEMMA 6.1. In the dqds algorithm with shift strategy $(\mathrm{C})$ the condition $0 \leq$ $s^{(n)}<\left(\sigma_{\min }^{(n)}\right)^{2}$ of $(9)$ is satisfied for all $n=0,1,2, \ldots$.

Proof. To prove the claim by induction on $n$, assume that

$$
0 \leq s^{(l)}<\left(\sigma_{\min }^{(l)}\right)^{2} \quad(l=0,1, \ldots, n-1) .
$$

If $\hat{d}_{k}^{(n+1)} \leq 0$ for some $k$ with $1 \leq k \leq m-1$, then $s^{(n)}=0$, which obviously satisfies (9). Therefore we may assume that $\hat{d}_{k}^{(n+1)}>0(k=1, \ldots, m-1)$ and the shift $s^{(n)}$ is given by (18). By Lemma 4.1 we have $q_{k}^{(n)}>0(k=1, \ldots, m)$ and $e_{k}^{(n)}>0(k=1, \ldots, m-1)$. Then, by Lemma 4.3 , the desired inequality $s^{(n)}<\left(\sigma_{\min }^{(n)}\right)^{2}$ holds if we have $d_{k}^{(n+1)}>0(k=1, \ldots, m)$ for $d_{k}^{(n+1)}$ computed by Algorithm 3.1 with the shift of (18). The latter is in fact true, as shown below. 
From the 3rd line (with $k=1$ ) of the shift strategy $(\mathrm{C})$ and the 3rd line of Algorithm 3.1 we have

$$
d_{1}^{(n+1)}-\hat{d}_{1}^{(n+1)}=q_{m}^{(n)}-s^{(n)} .
$$

By the 9th line of the shift strategy (C) we see

$$
q_{m}^{(n)}-s^{(n)}=\frac{e_{m-1}^{(n)} q_{m}^{(n)}}{\hat{d}_{m-1}^{(n+1)}+e_{m-1}^{(n)}}>0 .
$$

Hence, $d_{1}^{(n+1)}>\hat{d}_{1}^{(n+1)}$ is obtained. We can prove $d_{k}^{(n+1)}>\hat{d}_{k}^{(n+1)}(k=1, \ldots, m-1)$ by induction on $k$ as follows. By the 3rd line of the shift strategy (C) and (16) we have

$$
\begin{aligned}
d_{k}^{(n+1)}-\hat{d}_{k}^{(n+1)} & =\frac{d_{k-1}^{(n+1)} q_{k}^{(n)}}{d_{k-1}^{(n+1)}+e_{k-1}^{(n)}}-s^{(n)}-\left(\frac{\hat{d}_{k-1}^{(n+1)} q_{k}^{(n)}}{\hat{d}_{k-1}^{(n+1)}+e_{k-1}^{(n)}}-q_{m}^{(n)}\right) \\
& =\frac{e_{k-1}^{(n)} q_{k}^{(n)}\left(d_{k-1}^{(n+1)}-\hat{d}_{k-1}^{(n+1)}\right)}{\left(d_{k-1}^{(n+1)}+e_{k-1}^{(n)}\right)\left(\hat{d}_{k-1}^{(n+1)}+e_{k-1}^{(n)}\right)}+q_{m}^{(n)}-s^{(n)} .
\end{aligned}
$$

The induction hypothesis $d_{k-1}^{(n+1)}>\hat{d}_{k-1}^{(n+1)}$ together with $(20)$ implies $d_{k}^{(n+1)}>$ $\hat{d}_{k}^{(n+1)}$. Therefore $d_{k}^{(n+1)}>\hat{d}_{k}^{(n+1)}>0(k=1, \ldots, m-1)$ is proved. The remaining case with $k=m$ can be treated as follows. By (16) and (18) we have

$$
\begin{aligned}
d_{m}^{(n+1)} & =\frac{d_{m-1}^{(n+1)} q_{m}^{(n)}}{d_{m-1}^{(n+1)}+e_{m-1}^{(n)}}-\frac{\hat{d}_{m-1}^{(n+1)} q_{m}^{(n)}}{\hat{d}_{m-1}^{(n+1)}+e_{m-1}^{(n)}} \\
& =\frac{e_{m-1}^{(n)} q_{m}^{(n)}\left(d_{m-1}^{(n+1)}-\hat{d}_{m-1}^{(n+1)}\right)}{\left(d_{m-1}^{(n+1)}+e_{m-1}^{(n)}\right)\left(\hat{d}_{m-1}^{(n+1)}+e_{m-1}^{(n)}\right)} \\
& >0 .
\end{aligned}
$$

Thus we have proven $d_{k}^{(n+1)}>0(k=1, \ldots, m)$.

LEMMA 6.2. In the dqds algorithm with the shift strategy $(\mathrm{C})$ we have

$$
\lim _{n \rightarrow \infty} \hat{d}_{k}^{(n)}=\sigma_{k}^{2}-\sigma_{m}^{2}>0 \quad(k=1, \ldots, m-1),
$$

and hence $s^{(n)}=\hat{d}_{m-1}^{(n+1)} q_{m}^{(n)} /\left(\hat{d}_{m-1}^{(n+1)}+e_{m-1}^{(n)}\right)$ for all sufficiently large $n$.

Proof. By Lemma 6.1 Theorem 4.1 applies to our algorithm. In particular, $q_{k}^{(n)}$ and $e_{k}^{(n)}$ converge. We prove $(23)$ by induction on $k$. From the 3rd line of the shift strategy $(\mathrm{C})$ with $k=1$ we have

$$
\lim _{n \rightarrow \infty} \hat{d}_{1}^{(n+1)}=\lim _{n \rightarrow \infty}\left(q_{1}^{(n)}-q_{m}^{(n)}\right)=\sigma_{1}^{2}-\sigma_{m}^{2}>0
$$


which shows the case of $k=1$. From the 3rd line of the shift strategy (C) with general $k$ and the induction hypothesis we see

$$
\begin{aligned}
\lim _{n \rightarrow \infty} \hat{d}_{k+1}^{(n+1)} & =\lim _{n \rightarrow \infty}\left(\frac{\hat{d}_{k}^{(n+1)} q_{k+1}^{(n)}}{\hat{d}_{k}^{(n+1)}+e_{k}^{(n)}}-q_{m}^{(n)}\right) \\
& =\lim _{n \rightarrow \infty}\left(q_{k+1}^{(n)}-q_{m}^{(n)}\right) \\
& =\sigma_{k+1}^{2}-\sigma_{m}^{2} .
\end{aligned}
$$

Thus (23) holds true and therefore $\hat{d}_{k}^{(n+1)}>0(k=1, \ldots, m-1)$ for all sufficiently large $n$. As a result, the shift is determined as the 9 th line of the shift strategy $(\mathrm{C})$.

The limits of $q_{k}^{(n)}(k=1, \ldots, m)$ as $n \rightarrow \infty$ are given as follows.

LEMMA 6.3. In the dqds algorithm with the shift strategy $(\mathrm{C})$ we have

$$
\begin{aligned}
\sum_{n=0}^{\infty} s^{(n)} & =\sigma_{m}^{2}, \\
\lim _{n \rightarrow \infty} q_{k}^{(n)} & =\sigma_{k}^{2}-\sigma_{m}^{2} \quad(k=1, \ldots, m-1) ; \quad \lim _{n \rightarrow \infty} q_{m}^{(n)}=0 .
\end{aligned}
$$

Proof. By Lemma 6.2 we see

$$
\lim _{n \rightarrow \infty} s^{(n)}=\lim _{n \rightarrow \infty} \frac{\hat{d}_{m-1}^{(n+1)} q_{m}^{(n)}}{\hat{d}_{m-1}^{(n+1)}+e_{m-1}^{(n)}}=\lim _{n \rightarrow \infty} q_{m}^{(n)},
$$

whereas $\lim _{n \rightarrow \infty} s^{(n)}=0$ by (10) in Theorem 4.1. Hence $\lim _{n \rightarrow \infty} q_{m}^{(n)}=0$. This, together with (12), proves (24) and (25).

We can also identify the limits of $d_{k}^{(n)}(k=1, \ldots, m)$ as $n \rightarrow \infty$.

Lemma 6.4. In the dqds algorithm with the shift strategy $(\mathrm{C})$ we have

$$
\lim _{n \rightarrow \infty} d_{k}^{(n)}=\sigma_{k}^{2}-\sigma_{m}^{2} \quad(k=1, \ldots, m-1) ; \quad \lim _{n \rightarrow \infty} d_{m}^{(n)}=0 .
$$

Proof. This follows from Lemma 6.3 and the 5th line of Algorithm 3.1.

We now state the main theorem of this paper, which shows the cubic convergence of the dqds algorithm with the shift strategy $(\mathrm{C})$. In view of $(2)$ we introduce the notation

$$
r_{m}^{(n)}=q_{m}^{(n)}+\sum_{l=0}^{n-1} s^{(l)}-\sigma_{m}^{2}
$$

to represent the error in the approximated smallest eigenvalue of $B^{\mathrm{T}} B$. 
TheOREM 6.1 (Cubic convergence). In the dqds algorithm with the shift strategy (C) we have

$$
\begin{aligned}
& \lim _{n \rightarrow \infty} \frac{e_{m-1}^{(n+1)}}{\left(e_{m-1}^{(n)}\right)^{3}}=\frac{1}{\left(\sigma_{m-1}^{2}-\sigma_{m}^{2}\right)^{2}}, \\
& \lim _{n \rightarrow \infty} \frac{q_{m}^{(n+1)}}{\left(q_{m}^{(n)}\right)^{3}}=\frac{1}{\left(\sigma_{m-1}^{2}-\sigma_{m}^{2}\right)^{2}}, \\
& \lim _{n \rightarrow \infty} \frac{r_{m}^{(n+1)}}{\left(r_{m}^{(n)}\right)^{3}}=\frac{1}{\left(\sigma_{m-1}^{2}-\sigma_{m}^{2}\right)^{2}} .
\end{aligned}
$$

Therefore $e_{m-1}^{(n)}, q_{m}^{(n)}$ and $r_{m}^{(n)}$ are of cubic convergence. Moreover, we have

$$
\lim _{n \rightarrow \infty} \frac{r_{m}^{(n)}}{e_{m-1}^{(n)}}=0
$$

Proof. By Lemma 6.2 we may assume $s^{(n)}=\hat{d}_{m-1}^{(n+1)} q_{m}^{(n)} /\left(\hat{d}_{m-1}^{(n+1)}+e_{m-1}^{(n)}\right)$. From Algorithm 3.1 we see

$$
\begin{aligned}
\frac{e_{m-1}^{(n+2)}}{\left(e_{m-1}^{(n+1)}\right)^{3}} & =\frac{q_{m}^{(n+1)}}{\left(e_{m-1}^{(n+1)}\right)^{2} q_{m-1}^{(n+2)}} \\
& =\frac{d_{m}^{(n+1)}}{\left(e_{m-1}^{(n+1)}\right)^{2} q_{m-1}^{(n+2)}} \\
& =\frac{\left(q_{m-1}^{(n+1)}\right)^{2}}{q_{m-1}^{(n+2)}} \cdot \frac{d_{m}^{(n+1)}}{\left(e_{m-1}^{(n)} q_{m}^{(n)}\right)^{2}} \\
& =\frac{\left(q_{m-1}^{(n+1)}\right)^{2}}{q_{m-1}^{(n+2)}\left(d_{m-1}^{(n+1)}+e_{m-1}^{(n)}\right)\left(\hat{d}_{m-1}^{(n+1)}+e_{m-1}^{(n)}\right)} \cdot \frac{d_{m-1}^{(n+1)}-\hat{d}_{m-1}^{(n+1)}}{e_{m-1}^{(n)} q_{m}^{(n)}} \\
& =\frac{q_{m-1}^{(n+1)}}{q_{m-1}^{(n+2)}\left(\hat{d}_{m-1}^{(n+1)}+e_{m-1}^{(n)}\right)} \cdot \frac{d_{m-1}^{(n+1)}-\hat{d}_{m-1}^{(n+1)}}{e_{m-1}^{(n)} q_{m}^{(n)}},
\end{aligned}
$$

where the first equality is due to the 6 th line (with $k=m-1$ and $n$ replaced by $n+1$ ), the second is due to the 9 th line, the third is due to 6 th line (with $k=m-1$ ), the fourth is due to the second equality in (22), and the last is due to the 5th line (with $k=m-1$ ) of Algorithm 3.1.

In $(33)$ we have

$$
\lim _{n \rightarrow \infty} \frac{q_{m-1}^{(n+1)}}{q_{m-1}^{(n+2)}\left(\hat{d}_{m-1}^{(n+1)}+e_{m-1}^{(n)}\right)}=\frac{1}{\sigma_{m-1}^{2}-\sigma_{m}^{2}}
$$


by Lemmas 6.2 and 6.3. As for the other factor, we have, by (19) and (20),

$$
\begin{aligned}
\frac{d_{m-1}^{(n+1)}-\hat{d}_{m-1}^{(n+1)}}{e_{m-1}^{(n)} q_{m}^{(n)}} & =\frac{d_{1}^{(n+1)}-\hat{d}_{1}^{(n+1)}}{e_{m-1}^{(n)} q_{m}^{(n)}} \cdot \frac{d_{m-1}^{(n+1)}-\hat{d}_{m-1}^{(n+1)}}{d_{1}^{(n+1)}-\hat{d}_{1}^{(n+1)}} \\
& =\frac{1}{\hat{d}_{m-1}^{(n+1)}+e_{m-1}^{(n)}} \cdot \frac{d_{m-1}^{(n+1)}-\hat{d}_{m-1}^{(n+1)}}{d_{1}^{(n+1)}-\hat{d}_{1}^{(n+1)}}
\end{aligned}
$$

Here we have

$$
\lim _{n \rightarrow \infty}\left(\hat{d}_{m-1}^{(n+1)}+e_{m-1}^{(n)}\right)=\sigma_{m-1}^{2}-\sigma_{m}^{2}
$$

from (23) and also

$$
\lim _{n \rightarrow \infty} \frac{d_{m-1}^{(n+1)}-\hat{d}_{m-1}^{(n+1)}}{d_{1}^{(n+1)}-\hat{d}_{1}^{(n+1)}}=1,
$$

as is shown below. Then we obtain (29).

To prove (34) we show

$$
\lim _{n \rightarrow \infty} \frac{d_{k}^{(n+1)}-\hat{d}_{k}^{(n+1)}}{d_{1}^{(n+1)}-\hat{d}_{1}^{(n+1)}}=1 \quad(k=1, \ldots, m-1)
$$

by induction on $k$. The case of $k=1$ is obviously true. To treat the general case with $k$ we observe

$$
\frac{d_{k}^{(n+1)}-\hat{d}_{k}^{(n+1)}}{d_{1}^{(n+1)}-\hat{d}_{1}^{(n+1)}}=\frac{e_{k-1}^{(n)} q_{k}^{(n)}}{\left(d_{k-1}^{(n+1)}+e_{k-1}^{(n)}\right)\left(\hat{d}_{k-1}^{(n+1)}+e_{k-1}^{(n)}\right)} \cdot \frac{d_{k-1}^{(n+1)}-\hat{d}_{k-1}^{(n+1)}}{d_{1}^{(n+1)}-\hat{d}_{1}^{(n+1)}}+1
$$

from (19) and (21). Here we have $\lim _{n \rightarrow \infty} e_{k-1}^{(n)}=0$ by Lemma 6.1 and Theorem $4.1, \lim _{n \rightarrow \infty} q_{k}^{(n)}=\sigma_{k}^{2}-\sigma_{m}^{2}$ by Lemma $6.3, \lim _{n \rightarrow \infty} d_{k-1}^{(n+1)}=\lim _{n \rightarrow \infty} \hat{d}_{k-1}^{(n+1)}=$ $\sigma_{k-1}^{2}-\sigma_{m}^{2}$ by Lemmas 6.2 and 6.4 , and $\lim _{n \rightarrow \infty} \frac{d_{k-1}^{(n+1)}-\hat{d}_{k-1}^{(n+1)}}{d_{1}^{(n+1)}-\hat{d}_{1}^{(n+1)}}=1$ by the induction hypothesis. Thus we have proven (35).

Next we prove (30). From the 6th line (with $k=m-1$ ) of Algorithm 3.1, (29) and Lemma 6.3 we have

$$
\lim _{n \rightarrow \infty} \frac{q_{m}^{(n)}}{\left(e_{m-1}^{(n)}\right)^{2}}=\lim _{n \rightarrow \infty} \frac{q_{m-1}^{(n+1)} e_{m-1}^{(n+1)}}{\left(e_{m-1}^{(n)}\right)^{3}}=\frac{1}{\sigma_{m-1}^{2}-\sigma_{m}^{2}}
$$

and hence we obtain

$$
\lim _{n \rightarrow \infty} \frac{q_{m}^{(n+1)}}{\left(q_{m}^{(n)}\right)^{3}}=\lim _{n \rightarrow \infty}\left(\frac{e_{m-1}^{(n+1)}}{\left(e_{m-1}^{(n)}\right)^{3}}\right)^{2} \frac{q_{m}^{(n+1)} /\left(e_{m-1}^{(n+1)}\right)^{2}}{\left(q_{m}^{(n)} /\left(e_{m-1}^{(n)}\right)^{2}\right)^{3}}=\frac{1}{\left(\sigma_{m-1}^{2}-\sigma_{m}^{2}\right)^{2}} .
$$


Finally, we prove (31) and (32). From Algorithm 3.1 we have

$$
\begin{aligned}
q_{m}^{(n+1)} & =\frac{d_{m-1}^{(n+1)} q_{m}^{(n)}}{q_{m-1}^{(n+1)}}-s^{(n)} \\
& =\frac{\left(q_{m-1}^{(n+1)}-e_{m-1}^{(n)}\right) q_{m}^{(n)}}{q_{m-1}^{(n+1)}}-s^{(n)} \\
& =q_{m}^{(n)}-e_{m-1}^{(n+1)}-s^{(n)},
\end{aligned}
$$

where the first equality is due to the 9 th line and the 7 th line (with $k=m-1$ ), the second is due to the 5 th line, and the last is due to the 6th line. Adding both sides of the equation above over $n$ with $k=m$, we have

$$
q_{m}^{(n)}=q_{m}^{(0)}-\sum_{l=0}^{n-1} e_{m-1}^{(l+1)}-\sum_{l=0}^{n-1} s^{(l)} .
$$

Letting $n \rightarrow \infty$ and noting that $\sum_{l=0}^{\infty} e_{m-1}^{(l+1)}$ is convergent (see [1] for the detail), we also have

$$
q_{m}^{(\infty)}=q_{m}^{(0)}-\sum_{l=0}^{\infty} e_{m-1}^{(l+1)}-\sum_{l=0}^{\infty} s^{(l)},
$$

and hence, from (12) in Theorem 4.1,

$$
\sigma_{m}^{2}=q_{m}^{(\infty)}+\sum_{l=0}^{\infty} s^{(l)}=q_{m}^{(0)}-\sum_{l=0}^{\infty} e_{m-1}^{(l+1)} .
$$

Therefore, we see

$$
r_{m}^{(n)}=q_{m}^{(n)}+\sum_{l=0}^{n-1} s^{(l)}-\sigma_{m}^{2}=\sum_{l=n+1}^{\infty} e_{m-1}^{(l)} .
$$

It then follows from (29) that

$$
\lim _{n \rightarrow \infty} \frac{r_{m}^{(n)}}{e_{m-1}^{(n+1)}}=\lim _{n \rightarrow \infty} \frac{1}{e_{m-1}^{(n+1)}} \sum_{l=1}^{\infty} e_{m-1}^{(n+l)}=1 .
$$

Hence we obtain

$$
\begin{aligned}
\lim _{n \rightarrow \infty} \frac{r_{m}^{(n+1)}}{\left(r_{m}^{(n)}\right)^{3}} & =\lim _{n \rightarrow \infty} \frac{e_{m-1}^{(n+2)}}{\left(e_{m-1}^{(n+1)}\right)^{3}}=\frac{1}{\left(\sigma_{m-1}^{2}-\sigma_{m}^{2}\right)^{2}}, \\
\lim _{n \rightarrow \infty} \frac{r_{m}^{(n)}}{e_{m-1}^{(n)}} & =\lim _{n \rightarrow \infty} \frac{e_{m-1}^{(n+1)}}{e_{m-1}^{(n)}}=0
\end{aligned}
$$

from (29). 
Note that the critical variables for convergence are $e_{m-1}^{(n)}$ and $r_{m}^{(n)}$; the former is used for the convergence criterion and the latter represents the error in the approximation of $\sigma_{m}^{2}$. Furthermore, when the iteration is stopped at the $n$-th loop, the equation (32) indicates that $r_{m}^{(n)}$ is small enough compared to $e_{m-1}^{(n)}$. This property is useful in practice. Theorem 6.1 does not say anything about other variables, but this is already sufficient from the algorithmic point of view, since whenever the lower right elements, $e_{m-1}^{(n)}$ and $q_{m}^{(n)}$, converge to zero, the deflation is applied to reduce the matrix size.

REMARK 6.1. In the above, we intended to provide a streamlined rigorous proof of Theorem 6.1 by working directly with the recurrence relations in the dqds algorithm. Instead, we could have used a known identity for a shorter proof. In [15], the identity

$$
\begin{aligned}
q_{m}^{(n+1)}= & \left(e_{m-1}^{(n)} q_{m}^{(n)}\right)^{2}\left(\boldsymbol{v}^{(n)}\right)^{\mathrm{T}}\left(U^{(n)}-q_{m}^{(n)} I\right)^{-1} \boldsymbol{v}^{(n)} \\
& \times\left(\boldsymbol{v}^{(n)}\right)^{\mathrm{T}}\left(U^{(n)}-q_{m}^{(n)} I\right)^{-1}\left(U^{(n)}-\left(q_{m}^{(n)}+\hat{d}_{m}^{(n+1)}\right) I\right)^{-1} \boldsymbol{v}^{(n)}
\end{aligned}
$$

is shown. By the 6th line of Algorithm 3.1 with $k=m-1$, we then see

$$
\begin{aligned}
\frac{e_{m-1}^{(n+1)} q_{m}^{(n+1)}}{\left(e_{m-1}^{(n)} q_{m}^{(n)}\right)^{3}}= & \frac{1}{q_{m-1}^{(n+1)}}\left(\boldsymbol{v}^{(n)}\right)^{\mathrm{T}}\left(U^{(n)}-q_{m}^{(n)} I\right)^{-1} \boldsymbol{v}^{(n)} \\
& \times\left(\boldsymbol{v}^{(n)}\right)^{\mathrm{T}}\left(U^{(n)}-q_{m}^{(n)} I\right)^{-1}\left(U^{(n)}-\left(q_{m}^{(n)}+\hat{d}_{m}^{(n+1)}\right) I\right)^{-1} \boldsymbol{v}^{(n)}
\end{aligned}
$$

With this expression at hand, it is indeed immediate from Lemma 6.1 and Lemma 6.2 that

$$
\lim _{n \rightarrow \infty} \frac{e_{m-1}^{(n+1)} q_{m}^{(n+1)}}{\left(e_{m-1}^{(n)} q_{m}^{(n)}\right)^{3}}=\frac{1}{\left(\sigma_{m-1}^{2}-\sigma_{m}^{2}\right)^{4}},
$$

which shows the cubic convergence.

REMARK 6.2. The asymptotic cubic convergence claimed in this paper is surely the best rate ever known if the rate is defined against "steps" $(n)$. From the practical point of view, however, it is also natural to measure the convergence rate based on computational costs, and from this perspective the situation can be viewed in a slightly different manner. The shift strategy $(\mathrm{C})$ requires the computation of $\hat{d}_{k}^{(n+1)}$, generally for all of $k=1, \ldots, m-1$, whose computational cost is roughly $2 / 3$ of that of a dqds step, as noted in Remark 5.1. Thus, a full single step of the dqds with the shift strategy (C) requires costs $5 / 3$ times as much as the standard dqds, from which we may say that the actual rate of the "cubic" dqds is $3^{3 / 5} \simeq 1.93$. In this respect, the cubic dqds may fall behind, for example, the superquadratic one [2]. 


\section{Conclusion}

In this paper a concrete procedure for choosing the shifts in the dqds algorithm was proposed based on the shift strategy suggested by Rutishauser [15] and Fernando-Parlett [9]. Then it was proved with mathematical rigor that if the shift choosing procedure is employed the dqds always attains asymptotic cubic convergence for any initial bidiagonal matrix $B$.

Acknowledgments. Part of this work is supported by the 21st Century COE Program on Information Science and Technology Strategic Core and a Grant-in-Aid of the Ministry of Education, Culture, Sports, Science and Technology of Japan.

\section{References}

[1] K. Aishima, T. Matsuo, K. Murota and M. Sugihara, On Convergence of the dqds Algorithm for Singular Value Computation. SIAM Journal on Matrix Analysis and Applications, to appear.

[ 2 ] K. Aishima, T. Matsuo, K. Murota and M. Sugihara, A Shift Strategy for Superquadratic Convergence in the dqds Algorithm for Singular Values. Mathematical Engineering Technical Reports, METR 2007-12, University of Tokyo, March 2007.

[ 3 ] E. Anderson, Z. Bai, C. Bischof, S. Blackford, J. Demmel, J. Dongarra, J. Du Croz, A. Greenbaum, S. Hammarling, A. McKenny and D. Sorensen, LAPACK Users' Guide, Third Edition. SIAM, 1999.

[ 4 ] J. Demmel, Applied Numerical Linear Algebra. SIAM, Philadelphia, 1997.

[ 5 ] J. Demmel and W. Kahan, Accurate Singular Values of Bidiagonal Matrices. SIAM Journal on Scientific Computing, 11 (1990), 873-912.

[ 6 ] I.S. Dhillon, A New $O\left(n^{2}\right)$ Algorithm for the Symmetric Tridiagonal Eigenvalue/Eigenvector Problem. Ph.D. Thesis, Computer Science Division, University of California, Berkeley, California, 1997.

[ 7 ] I.S. Dhillon and B.N. Parlett, Multiple Representations to Compute Orthogonal Eigenvectors of Symmetric Tridiagonal Matrices. Linear Algebra and Its Applications, 387 (2004), $1-28$.

[ 8 ] I.S. Dhillon and B.N. Parlett, Orthogonal Eigenvectors and Relative Gaps. SIAM Journal on Matrix Analysis and Applications, 25 (2004), 858-899.

[ 9 ] K.V. Fernando and B.N. Parlett, Accurate Singular Values and Differential qd Algorithms. Numerische Mathematik, 67 (1994), 191-229.

[10] P. Henrici, Applied and Computational Complex Analysis, Vol. 1. Wiley, New York, 1974.

[11] LAPACK, http://www.netlib.org/lapack/.

[12] B.N. Parlett, The New qd Algorithm. Acta Numerica, 1995, 459-491.

[13] B.N. Parlett, The Symmetric Eigenvalue Problem. Prentice-Hall, Englewood Cliffs, New Jersey, 1980; SIAM, Philadelphia, 1998.

[14] B.N. Parlett and O. Marques, An Implementation of the dqds Algorithm (Positive Case). Linear Algebra and Its Applications, 309 (2000), 217-259.

[15] H. Rutishauser, Über eine kubisch konvergente Variante der LR-Transformation. Zeitschrift für Angewandte Mathematik und Mechanik, 11 (1960), 49-54.

[16] J.H. Wilkinson, The Algebraic Eigenvalue Problem. Clarendon Press, Oxford, 1965. 
